\title{
Characterization of two putative potassium channels in Plasmodium falciparum
}

\author{
Karena L Waller ${ }^{1,5}$, Sean M McBride ${ }^{2}$, Kami Kim*3,4 and \\ Thomas V McDonald*1,2
}

\begin{abstract}
Address: ${ }^{1}$ Department of Medicine (Cardiology), Albert Einstein College of Medicine, 1300 Morris Park Ave., Bronx NY 10461, USA, ${ }^{2}$ Department of Molecular Pharmacology, Albert Einstein College of Medicine, Bronx NY 10461, USA, ${ }^{3}$ Department of Medicine (Infectious Diseases), Albert Einstein College of Medicine, Bronx NY 10461, USA, ${ }^{4}$ Department of Microbiology and Immunology, Albert Einstein College of Medicine, Bronx NY 10461, USA and ${ }^{5}$ Department of Microbiology, Monash University, Clayton VIC 3800, Australia
\end{abstract}

Email: Karena LWaller - karena.waller@med.monash.edu.au; Sean M McBride - smcbride@aecom.edu.au; Kami Kim* - kkim@aecom.yu.edu; Thomas V McDonald* - mcdonald@aecom.yu.edu

* Corresponding authors

Published: 24 January 2008

Malaria Journal 2008, 7:19 doi:10.1186/1475-2875-7-19

This article is available from: http://www.malariajournal.com/content/7/I//9

(C) 2008 Waller et al; licensee BioMed Central Ltd.

This is an Open Access article distributed under the terms of the Creative Commons Attribution License (http://creativecommons.org/licenses/by/2.0), which permits unrestricted use, distribution, and reproduction in any medium, provided the original work is properly cited.
Received: 26 October 2007

Accepted: 24 January 2008

\begin{abstract}
Background: Potassium channels are essential for cell survival and participate in the regulation of cell membrane potential and electrochemical gradients. During its lifecycle, Plasmodium falciparum parasites must successfully traverse widely diverse environmental milieus, in which $\mathrm{K}^{+}$channel function is likely to be critical. Dramatically differing conditions will be presented to the parasite in the mosquito mid-gut, red blood cell (RBC) cytosol and the human circulatory system.

Methods: In silico sequence analyses identified two open-reading frames in the $P$. falciparum genome that are predicted to encode for proteins with high homology to $\mathrm{K}^{+}$channels. To further analyse these putative channels, specific antisera were generated and used in immunoblot and immunofluorescence analyses of $P$. falciparum-infected RBCs. Recombinant genome methods in cultured $P$. falciparum were used to create genetic knock outs of each $\mathrm{K}^{+}$channel gene to asses the importance of their expression.

Results: Immunoblot and IFA analyses confirmed the expression of the two putative $P$. falciparum $\mathrm{K}^{+}$channels (PfKI and PfK2). PfKI is expressed in all asexual stage parasites, predominantly in late stages and localizes to the RBC membrane. Conversely, PfK2 is predominantly expressed in late schizont and merozoite stage parasites and remains primarily localized to the parasite. Repeated attempts to knockout PfKI and PfK2 expression by targeted gene disruption proved unsuccessful despite evidence of recombinant gene integration, indicating that $p f k I$ and $p f k 2$ are apparently refractory to genetic disruption.

Conclusion: Putative $\mathrm{K}^{+}$channel proteins $\mathrm{PfKI}$ and $\mathrm{PfK} 2$ are expressed in cultured $P$. falciparum parasites with differing spatial and temporal patterns. Eventual functional characterization of these channels may reveal future pharmacological targets.
\end{abstract}




\section{Background}

In the course of a persistent human infection, Plasmodium falciparum parasites are exposed to dramatic changes in the extracellular milieu in terms of $\mathrm{pH}$, osmolarity and ionic constituents. To survive such conditions transporter pumps, exchangers and ion channels operate to maintain the intra-parasite environment in the face of varying external conditions. Many classes of putative $P$. falciparum channels and transporters have been identified [1]. These are thought to be critical for parasite viability $[2,3]$ and offer potential new molecular targets for the development of novel anti-malarials.

A

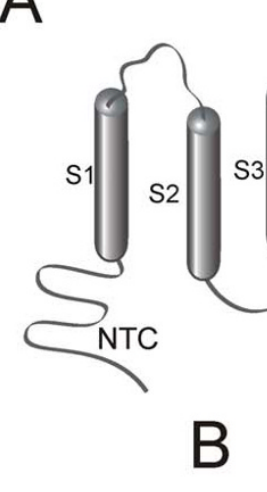

One class of transporters, the potassium $\left(\mathrm{K}^{+}\right)$channels, are transmembrane proteins that gate open and closed to control the flow of $\mathrm{K}^{+}$ions across cell membranes. $\mathrm{K}^{+}$ channels are critical in the regulation of the transmembrane electrochemical gradient, cell membrane potential and intracellular osmolarity and are essential for the survival of all known cells. $\mathrm{K}^{+}$channels share several common features. They have 2, 4 or 6 membrane-spanning regions, two of which contribute to form the "pore region" through which $\mathrm{K}^{+}$ions move (Figure $1 \mathrm{~A}$ ). A highly conserved GYG (or the less common GFG) signature motif within the pore region comprises the ion selectivity filter [4]. Functional channels result from a tetrameric
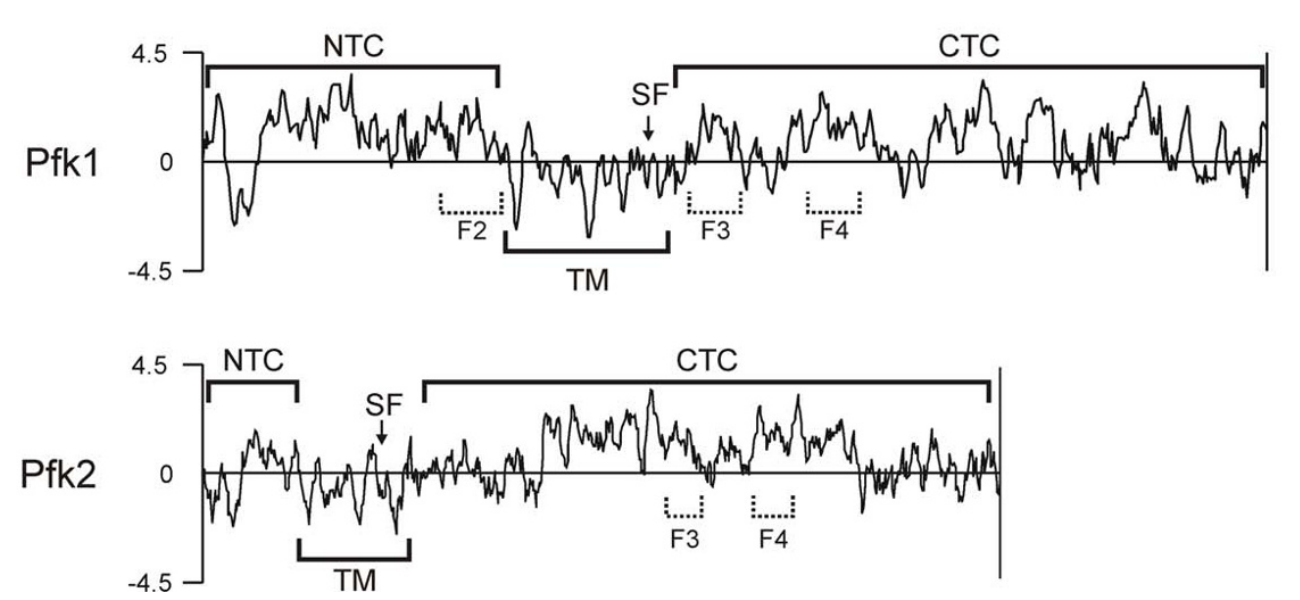

Figure I

PfKI and PfK2. A. Membrane topology schematic of a single $\mathrm{K}^{+}$channel subunit. Predicted topographical domains are indicated: NTC = N-terminal cytoplasmic portion; CTC = C-terminal cytoplasmic portion; SF = selectivity filter; and SI-S6 = membrane spanning domains. B. Hydropathy plots of the predicted protein sequences of PfKI and PfK2. Negative deflections signify hydrophobic sequences. NTC $=\mathrm{N}$-terminal cytoplasmic portion; $\mathrm{CTC}=\mathrm{C}$-terminal cytoplasmic portion; TM $=$ Transmembrane portion and SF = selectivity filter. F2, F3 and F4 refer to protein segments used to generate specific antisera. $\mathbf{C}$. Alignment of channel conduction pore protein sequences of various well characterized human $\mathrm{K}^{+}$channels, highlighting features common to the family $\left(\mathbf{G}=\right.$ suggested flexibility point for voltage-gated $\mathrm{K}^{+}$channels. $\mathbf{P}=$ possible bend-inducing prolines. $\mathbf{F}=$ sites for possible drug block). Kvl.4 = voltage-gated Shaker-type $\mathrm{K}^{+}$channel, Kir2.I = inward rectifying $\mathrm{K}^{+}$channel, hERG = human Ether a-gogo-Related Gene channel, huBK = human BK Ca ${ }^{2+}$-activated $\mathrm{K}^{+}$channel, Gardos IK K ${ }^{+}$channel and SKCa3 SK $\mathrm{Ca}^{2+}$-activated $\mathrm{K}^{+}$channel. The sequence encoded by PFI4_0342 is also included in the alignment, due to its suggestion as being a possible third parasite-encoded $\mathrm{K}^{+}$channel [I]. PFI4_0342 does not have several features common to $\mathrm{K}^{+}$channels, including the pore region GYG motif. 
Table I: Oligonucleotide primers.

\begin{tabular}{|c|c|c|}
\hline Primer & Sequence $\left(5^{\prime} \rightarrow 3^{\prime}\right)^{a}$ & Target $^{b}$ \\
\hline $\mathrm{PI}$ & cgggaattcATGAATAATGATAATATTGGGAG & $p f k /$ full length $(+)$ \\
\hline P2 & cccaagcttTCAGACTTGGTCATGCGGTTC & pfk I full length (-) \\
\hline P3 & cgggaattcGTTGATAAGAAAGGAAAAATAATAGAT & $p f k / F 2(+)$ \\
\hline P4 & cccaagcttATGTACAAATCGATGTTTTGTATA & $p f k I F 2(-)$ \\
\hline P5 & ccgggaattcatTTTAATAATCATGATCATCAGAATC & pfkl F3 (+) \\
\hline P6 & cccaagcttTAAACATTCTTGATCATCTTTTTCC & $p f k / F 3(-)$ \\
\hline P7 & ccgggaattctcAAAAAAGGACAAAACCATCAACTT & $\mathrm{pfk} / \mathrm{F} 4(+)$ \\
\hline P8 & cccaagcttCATATTTTTTTCCATATCTTTAATG & $p f k / F 4(-)$ \\
\hline $\mathrm{Pg}$ & cgcggatccgATGAAAAGCGGATTATTTTCTATG & $p f k 2$ full length $(+)$ \\
\hline PIO & ccggaattcTCACAATATATAAACTATATCATCGAATC & $p f k 2$ full length (-) \\
\hline PII & ccgggaattcATAATGATAATGATAATAATAATATTATA & $p f k 2 \mathrm{~F} 3(+)$ \\
\hline $\mathrm{P} 12$ & cccaagctttTTTAATTCCTTTTTATTATCAATTGT & pfk2 F3 (-) \\
\hline $\mathrm{PI3}$ & ccgggaattcGAATATCAAGAATATTTACCAAAAAC & pfk2 F4 (+) \\
\hline $\mathrm{PI} 14$ & cccaagcttATTATGAATATGAATATTTGTATTTGA & pfk2 F4 (-) \\
\hline $\mathrm{PI5}$ & aaactgcagTGAAAACAGAATGCGATTAGTTCG & $\mathrm{pfk} / \mathrm{KO}(+)$ \\
\hline $\mathrm{PI} 6$ & cgcggatccGATGATGCAAATGTTAATGGTAC & $p f k I$ KO (-) \\
\hline $\mathrm{PI7}$ & aaactgcagTTCTTATCTTATTGAACGGATCTC & $\mathrm{pfk} 2 \mathrm{KO}(+)$ \\
\hline $\mathrm{P} 18$ & cgcggatccTTGTATGGTATATATAGTAGATGC & pfk2 KO (-) \\
\hline PI9 & GATAGCGATTTTTTTTACTGTCTG & hrp2 3'UTR (+) \\
\hline $\mathrm{P} 20$ & GAAATTAACCCTCACTAAAGGG & plasmid backbone \\
\hline P2I & aaggaaaaaagcggccgcAATGAATAATGATAATATTGGGAG & $p f k l(+)$ \\
\hline $\mathrm{P} 22$ & cccaagcttAGATATAATACCATCCGTTTGG & $p f k I(-)$ \\
\hline $\mathrm{P} 23$ & ccggaattcAATGAAAAGCGGATTATTTTCTATG & $p f k 2(+)$ \\
\hline P24 & cgcggatccTTGTATGGTATATATAGTAGATGC & $p f k 2(-)$ \\
\hline P25 & TTGTTGTGGTTCTACATCTCC & pfk2 (-) \\
\hline $\mathrm{P} 26$ & CACGAAGCCGCCACACATTGCC & hrp2 orfl \\
\hline $\mathrm{P} 27$ & TTTGATCTTGTTCACTATGGC & $p f k l(-)$ \\
\hline $\mathrm{P} 28$ & cccaagcttATTACAAAAATAATAATTGAAATTACT & $p f k 2(-)$ \\
\hline P29 & GTAATACGACTCACTATAGGGC & plasmid backbone \\
\hline P30 & TTGCAATTCTGCTTCAGTTGG & $\operatorname{cam}(-)$ \\
\hline
\end{tabular}

\footnotetext{
a Sequences shown in upper case are gene specific. Underlined sequences indicate enzyme restriction sites.

$b+$ and - indicate sense and anti-sense strand sequences, respectively.
}

complex of identical or closely related subunits, with the ion conduction pathway formed by the four-fold symmetry of the component subunits.

Here, a bioinformatic approach was used to identify two putative $\mathrm{K}^{+}$channel genes ( $p f k 1$ and $p f k 2$ ). This report details the cellular investigation of these putative $\mathrm{K}^{+}$channel proteins, showing their differential expression and cellular localization in asexual blood stage parasites. The identification of only two putative $\mathrm{K}^{+}$channels encoded within the $P$. falciparum genome, in conjunction with their differing expression and localization profiles suggests that these proteins may play unique and potentially critical roles in the maintenance of parasite viability.

\section{Methods}

\section{Parasite culture}

Plasmodium falciparum 3D7 parasites were maintained by in vitro culture using standard culture conditions [5] and supplemented with 0.5\% Albumax II (Invitrogen). Parasi- taemias were determined by microscopic examination of Giemsa stained thin blood smears.

\section{Homology searches, molecular coning and knockout parasite transfection}

Homology searches of the $P$. falciparum 3D7 genome database [6] were performed using the conserved $\mathrm{K}^{+}$channel GYG selectivity filter motif. Extracted sequences were further scrutinized to identify any predicted flanking membrane-spanning segments. Two gene sequences, designated $p f k 1$ (PFL1315w) and $p f k 2$ (PF14_0622), were identified and amplified from genomic $P$. falciparum 3D7 DNA by PCR using specific primers (Table 1). All nucleotide sequences were confirmed by automated sequence analysis (Genewiz Inc.).

Approximately $1.0 \mathrm{~kb}$ regions of $p f k 1$ and $p f k 2$ (downstream of, but not including the endogenous ATG start site) were amplified by PCR (Table 1) and cloned into the transfection plasmid pminiBSD [7], generating the plasmids pminiBSD/pfk1-KO and pminiBSD/pfk2-KO. Trans- 
fection of $P$. falciparum 3D7 parasites was performed using previously described methods $[8,9]$. Transfected cultures were screened by PCR for plasmid integration [10] using specific primers (Table 1) approximately three months after transfection. Integration positive cultures were then cloned by limiting dilution [10].

\section{Western blotting and indirect immunofluorescence assays (IFA)}

Recombinant Glutathione S-transferase (GST) fusion proteins of PfK1 and PfK2 were generated. Regions of $p f k 1$ and $p f k 2$ (Figure 1B) were cloned into pGEX-KG and the encoded fusion proteins expressed and purified using standard affinity chromatography [11]. Rabbit polyclonal antibodies were generated (Covance), and affinity purification was performed using the GST-fusion proteins immobilized on CN-Br Sepharose (Amersham Biosciences). The paired pre-immune control sera were obtained prior to immunization of the rabbits, and were used in simultaneous immunochemical methodologies (see above) as controls. No cross-reactivity was observed.

Parasite lysates were prepared from cultures synchronized by sorbitol lysis [12]. Cultures were sampled at regular intervals and the cells lysed using $0.15 \%$ saponin in phosphate-buffered saline (PBS; $137 \mathrm{mM} \mathrm{NaCl}, 2.7 \mathrm{mM} \mathrm{KCl}$, $\left.4.3 \mathrm{mM} \mathrm{Na}_{2} \mathrm{HPO}_{4}, 1.4 \mathrm{mM} \mathrm{KH} \mathrm{PO}_{4}, \mathrm{pH} .4\right)$. Parasites were washed in STE $(100 \mathrm{mM} \mathrm{NaCl}, 20 \mathrm{mM}$ Tris-HCl pH8.0, $50 \mathrm{mM}$ EDTA), before resuspending in reducing SDS-PAGE loading buffer and incubation at $37^{\circ} \mathrm{C}$ for 30 minutes. Samples were not boiled to facilitate solubility and prevent aggregation of transmembrane proteins $[13,14]$. Proteins were resolved in $7.5 \%$ or $4-15 \%$ gradient polyacrylamide gels (Bio-Rad Laboratories) before transfer to nitrocellulose membranes. Western blotting was performed using affinity purified anti-PfK1 or antiPfK2 antibodies (1/500) and detected using Western Lightning Chemiluminescence Reagent Plus (PerkinElmer Life Sciences). Control western blots were performed using polyclonal anti-KAHRP (provided by R. L. Coppel) and anti-GRP(BiP) antisera ([15]; MRA20, MR4 ATCC).

Cellular localization of PfK1 and PfK2 by IFAs was performed on sorbitol-synchronized parasites. Thin blood smears were prepared from cultures at approximately 12hour intervals before fixation with methanol/acetone $(9: 1)$. Slides were incubated with affinity purified antibodies in PBS containing $0.5 \%$ Bovine Serum Albumin (BSA) for 30 minutes, washed extensively with PBS and stained with goat anti-rabbit AlexaFluor ${ }^{\circledR} 88$ conjugate antibody (1/1000; Molecular Probes). For doublelabelled IFAs, thin blood smears were prepared in the same manner and sequentially incubated with primary antibodies before staining with goat anti-rabbit AlexaFluor $^{\circledR} 488$ and goat anti-mouse AlexaFluor ${ }^{\circledR} 594$ conju- gate antibodies (both 1/1000; Molecular Probes). Parasite nuclei were visualized with 4',6-Diamidino-2-phenylinodole dihydrochloride (DAPI; $10 \mu \mathrm{g} / \mathrm{ml}$ ). Slides were washed and mounted in Fluoromount-G (Southern Biotech). Cells were visualized using an Olympus IX81 electronically motorized microscope. Images were captured using a Sensicam QE cooled CCD camera (The Cooke Corporation) and IP Lab Spectrum Scientific Imaging Processing Software (Scanalytics Inc.). Images were then deconvolved using Vaytek Image software (Vaytek Inc.). The primary antibodies were: affinity purified anti-PfK1 F4 (1/250) and anti-PfK2 F4 (1/100), polyclonal rabbit anti-KAHRP (1/250) and anti-MESA (1/250 [11] ; provided by R. L. Coppel) and mouse mAb5.2 anti-MSP1 (1/ 100; MRA94, MR4 ATCC).

\section{Results \\ Identification of pfk I and pfk2}

Searches of the P. falciparum 3D7 genome database using the conserved $\mathrm{K}^{+}$channel selectivity filter GYG motif yielded two gene sequences, designated here $p f k 1$ (PFL1315w, or $p f k c h 1[16]$ ) and $p f k 2$ (PF14_0622). Pfk1 is approximately $6.1 \mathrm{~kb}$ and is located on chromosome 12 . The pfk2 gene is approximately $4.4 \mathrm{~kb}$ and located on chromosome 14 . Both genes possess a single exon and are predicted to encode highly charged proteins; PfK1 is rich in asparagine, lysine, arginine, glutamic and aspartic acid, whereas $\mathrm{PfK} 2$ is rich in asparagine, lysine, leucine, isoleucine and tyrosine. Both protein sequences are predicted to have six membrane-spanning regions and a pore region (Fig. 1B). Alignments of the pore region sequence from PfK1 and PfK2 with other well characterized $\mathrm{K}^{+}$channels demonstrates overall similarity between these $\mathrm{K}^{+}$channel proteins (Figure 1C), with closest similarity observed within the pore region of $\mathrm{Ca}^{2+}$-activated $\mathrm{K}^{+}$channels.

\section{Differential expression of PfKI and PfK2}

Immunoblots of synchronized parasites were performed using affinity purified antibodies specific for PfK1 and PfK2 (Figure 2A) and pre-immune control sera (no crossreactivity was observed). Initially, three different antiPfK1 and two anti-PfK2 antibodies were used to probe identical synchronized parasite samples. In each case, PfK1 expression was detected as a group of three high molecular mass bands of $>200 \mathrm{kDa}$, whereas PfK2 expression was detected as a doublet of $>250 \mathrm{kDa}$ band (Figure 2A). Further, immunoblots of time-course parasite material showed PfK1 was expressed throughout the $48 \mathrm{hr}$ asexual lifecycle, but greater expression levels were detected in more mature parasites. PfK2 abundance also varied through the $48 \mathrm{hr}$ time course, with maximal expression being detected in samples rich in merozoites and late schizonts (Figure 2B). The multiple bands of PfK1 and PfK2 may be attributed to incomplete disruption of PfK oligomers into individual subunits or from associa- 
tion of other $\mathrm{K}^{+}$channel regulatory subunits during the relatively mild sample preparation conditions.

Additional blots were performed in parallel using antibodies against the endoplasmic reticulum (ER) marker protein Glucose-regulated protein GRP(BiP) [15] and the Knob Associated Histidine Rich Protein (KAHRP; Fig. 2). GRP(BiP) mRNA has been shown by microarray to be constitutively expressed across the $48 \mathrm{hr}$ asexual life cycle [17-19]. Conversely, KAHRP is expressed by intracellular parasites in a stage-specific manner (see Cooke et al for review [20]). GRP(BiP) was readily detected in all samples at approximately equal levels, whereas maximal KAHRP expression was detected in mature trophozoites and schizonts.

\section{Differential localization of PfKI and PfK2}

Although detectable by Western blotting, PfK1 was not detected by IFA in ring infected red blood cells (iRBCs) or in surrounding uninfected red blood cells (uRBCs; Figure 3). However, IFA labeling was detected in trophozoites and schizonts (using anti-PfK1 F4 antibodies). The level of labeling increased with parasite maturity and was located at the plasma membrane of the iRBC, in a semipunctate pattern (Figure 3A). Intense punctate labeling of PfK2 was detected in mature schizonts using anti-PfK2 F4 antibodies, but not in uRBCs (Figure 3B), in a pattern suggesting co-localization with the developing merozoites within the schizont. Anti-PfK2 F4 antibodies failed to label rings, with minimal labeling detected in trophozoites. Similar IFA results for both PfK1 and PfK2 localization were obtained using the alternate antibodies antiPfK1 F3 and anti-PfK2 F3, respectively, and pre-immune control sera showed not cross-reactivity.

Control IFAs were performed with KAHRP and Mature parasite-infected Erythrocyte Surface Antigen (MESA) antisera. KAHRP is exported into the red blood cell (RBC) cytosol where it associates with the membrane skeleton, forming electron-dense, knob-like structures at the RBC membrane. MESA is exported from the intracellular parasite into the RBC cytosol where it associates with the membrane skeleton, but has no specific association with knobs (see Cooke et al for review [20]). Increasing intensity of punctate KAHRP labeling was observed with increasing parasite maturity, with maximal labeling detected on the RBC membrane of trophozoites and schizonts (Figure 3C). Increased MESA labeling was observed at the RBC membrane of the mature trophozoites and schizonts with a nearly confluent appearance (Figure 3D). The pattern of PfK1 labeling more closely resembled the punctate appearance of the RBC membrane-associated protein KAHRP, rather than that of MESA.

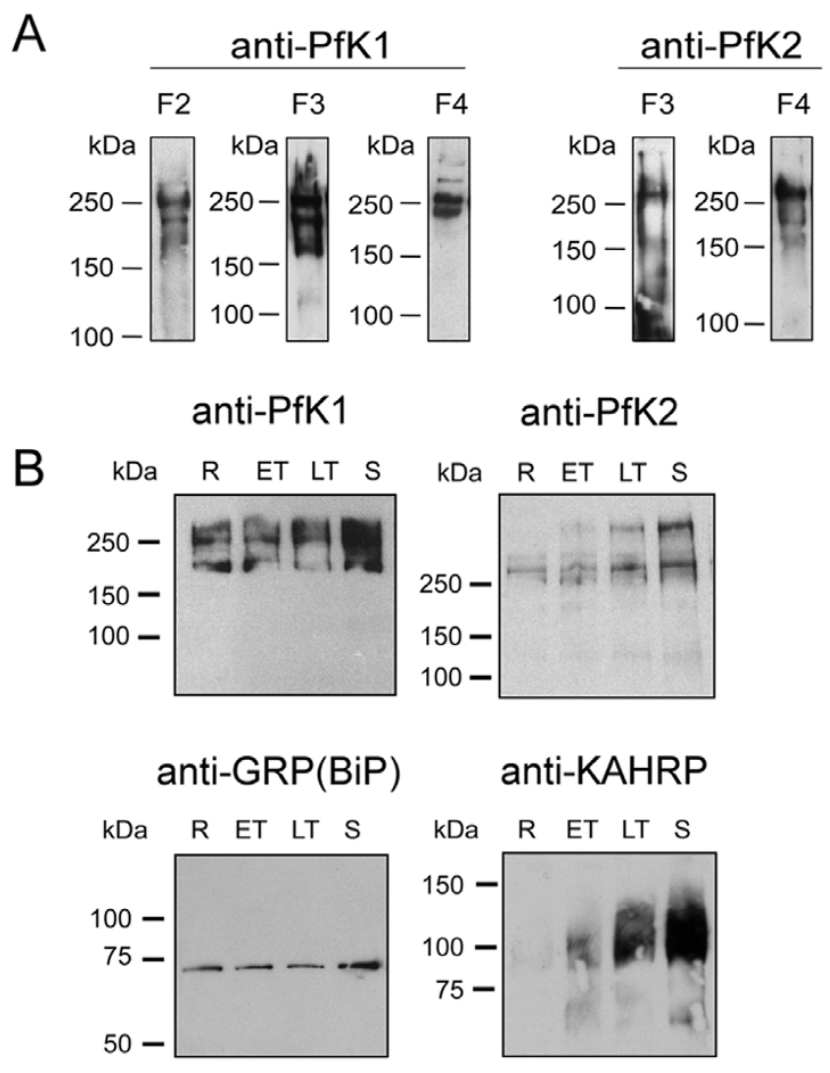

Figure 2

Expression of PfKI and PfK2. A. Immunoblots of identical synchronized parasite samples (loading $2 \times 10^{7}$ parasites per lane; rich in schizonts and early rings) probed with each of the three anti-PfKI and two anti-PfK2 antibodies. Similar banding profiles are detected by each anti-PfKI or anti-PfK2 antibodies. B. Synchronous cultures were sampled over the $48 \mathrm{hr}$ lifecycle and probed by Western Blot (loading $\sim 1 \times 10^{7}$ parasites per lane). PfKI was detected as a triplet set of bands of $>200 \mathrm{kDa}$ using anti-PfK I F3 antibodies. Expression of PfKI was detected across the $48 \mathrm{hr}$ lifecycle. PfK2 was detected as two bands of $>250 \mathrm{kDa}$ using anti-PfK $2 \mathrm{~F} 4$ antibodies, with maximal expression detected in late schizonts (ie. samples rich in developing merozoites). Constitutive expression of the ER marker protein GRP(BiP) across the 48 $\mathrm{hr}$ lifecycle of $P$. falciparum was detected at approximately 70 $\mathrm{kDa}$. Stage specific expression of KAHRP, observed as a broad band of reactivity at $\sim 100 \mathrm{kDa}$, with maximal expression observed in mature trophozoites and schizonts. Minimal expression was detected in samples rich in ring stage parasites. The samples are: rings (R), early trophozoites (ET), late trophozoites (LT) and schizonts (S).

To further define the localization of PfK2, double-labeled IFAs using antibodies against the merozoite markers MSP1 (Figure 4) and MSP4 were performed. The most intense staining suggests that PfK2 is associated directly with the parasite. In schizonts, labeling was primarily confined to the parasite itself, with small intensely labeled 


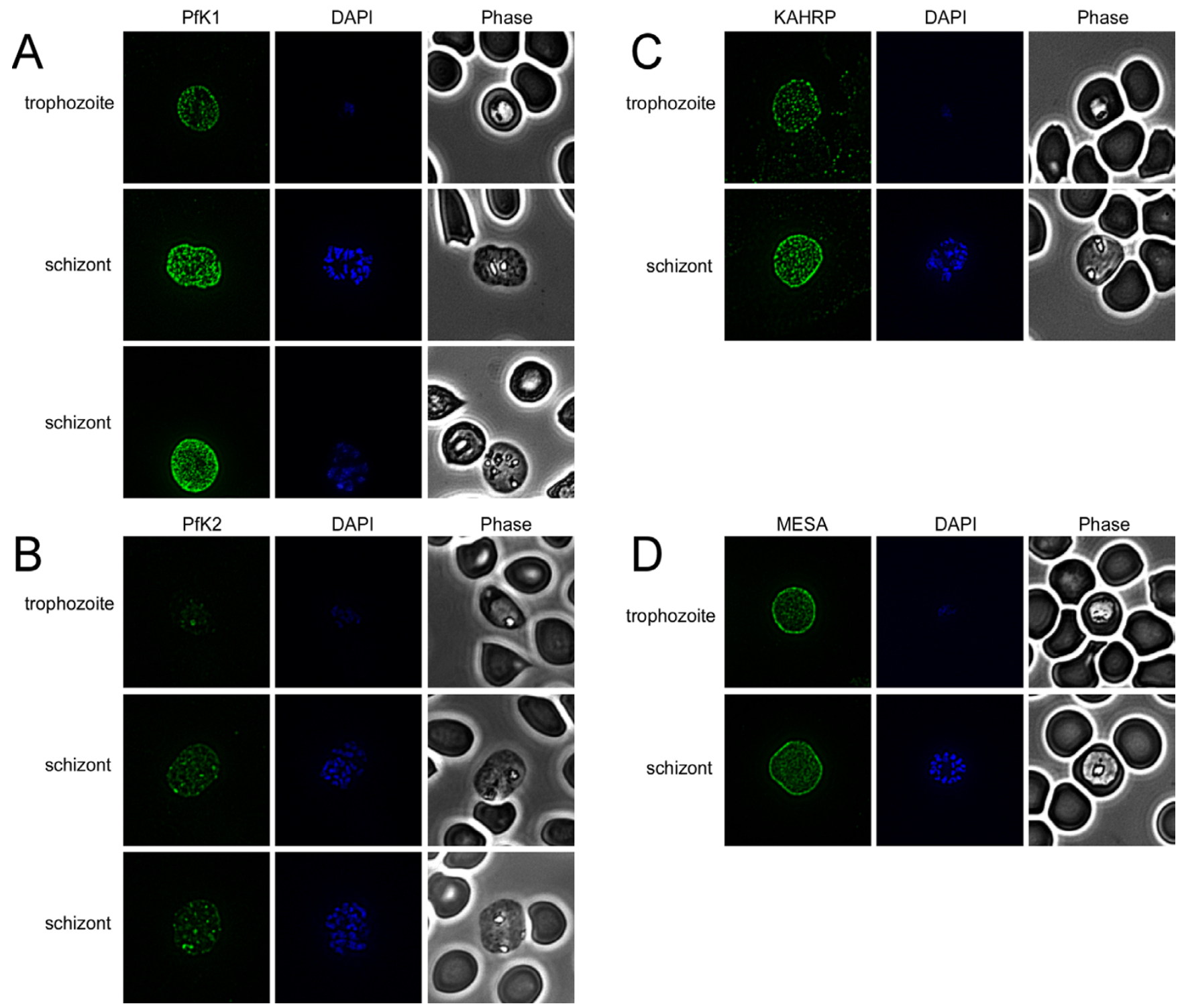

Figure 3

Localization of PfKI and PfK2. Indirect Immunofluorescence Assays (IFAs) were performed using affinity purified anti-PfKI F4 and -PfK2 F4 antibodies, and anti-KAHRP and anti-MESA antisera. Representative IFAs are shown for each antibody. A Anti.-PfKI IFAs showed no labeling in rings (data not shown) or uRBCs, and increasing RBC membrane labeling in trophozoites and schizonts. Predominantly labeling occurs in a semi-punctate localization pattern. B. PfK2 was not detected in rings (data not shown) or uRBCs, and minimal levels were detected in trophozoites. Schizonts showed intense foci of labeling, which appeared to correspond with the location of the developing merozoites within RBCs. C. KAHRP was detected in a classic punctuate pattern on the surface of trophozoites and schizonts. No labeling of KAHRP was detected in ring-iRBCs (data not shown). D. More diffuse RBC membrane labeling of MESA was detected on trophozoites and schizonts. No labeling was detected in rings (data not shown).

regions appearing to co-localize with developing merozoites. Lower levels of diffuse labeling of PfK2 in intermerozoite regions and $\mathrm{RBC}$ cytoplasm were also detected in some iRBCs. However, resolution by IFA was not sufficient to definitively determine whether there was RBC membrane association. Numerous localization attempts using immuno-electron microscopy failed with affinity purified antibodies specific for both PfK1 and PfK2.

\section{Pfkl and pfk2 knockout (KO) transfections}

Genetic disruption or knockout (KO) of $p f k 1$ and $p f k 2$ expression was attempted to examine the importance of 
these $\mathrm{K}^{+}$channels in parasite viability in vitro. After transfection, PCRs were performed to demonstrate the presence of $\mathrm{KO}$ disrupted $p f k 1$ and $p f k 2$ parasites in transfection cultures (Figure 5). In each case, PCR amplification of the expected size DNA products demonstrated integration into both the $p f k 1$ or $p f k 2$ loci in bulk transfection cultures (Figure 5, panels B and C, lanes d1 and d2), but despite repeated attempts, no clones were isolated after cloning by limited dilution. That $p f k 1$ and $p f k 2 \mathrm{KO}$ integration can be detected via PCR in bulk transfection cultures, but a parasite clone could not be isolated sug- gests that disruption of these genes is deleterious to parasite viability.

\section{Discussion}

Asexual $P$. falciparum parasites must rapidly respond to the widely variant ionic conditions, $\mathrm{pH}$ and osmolarities of their external milieus. In order to adapt to these environments, parasites most likely utilize ion channels and transporters to facilitate their regulation of intracellular ion concentrations. Allen and Kirk have shown that the transmembrane potential across the Plasmodium parasite

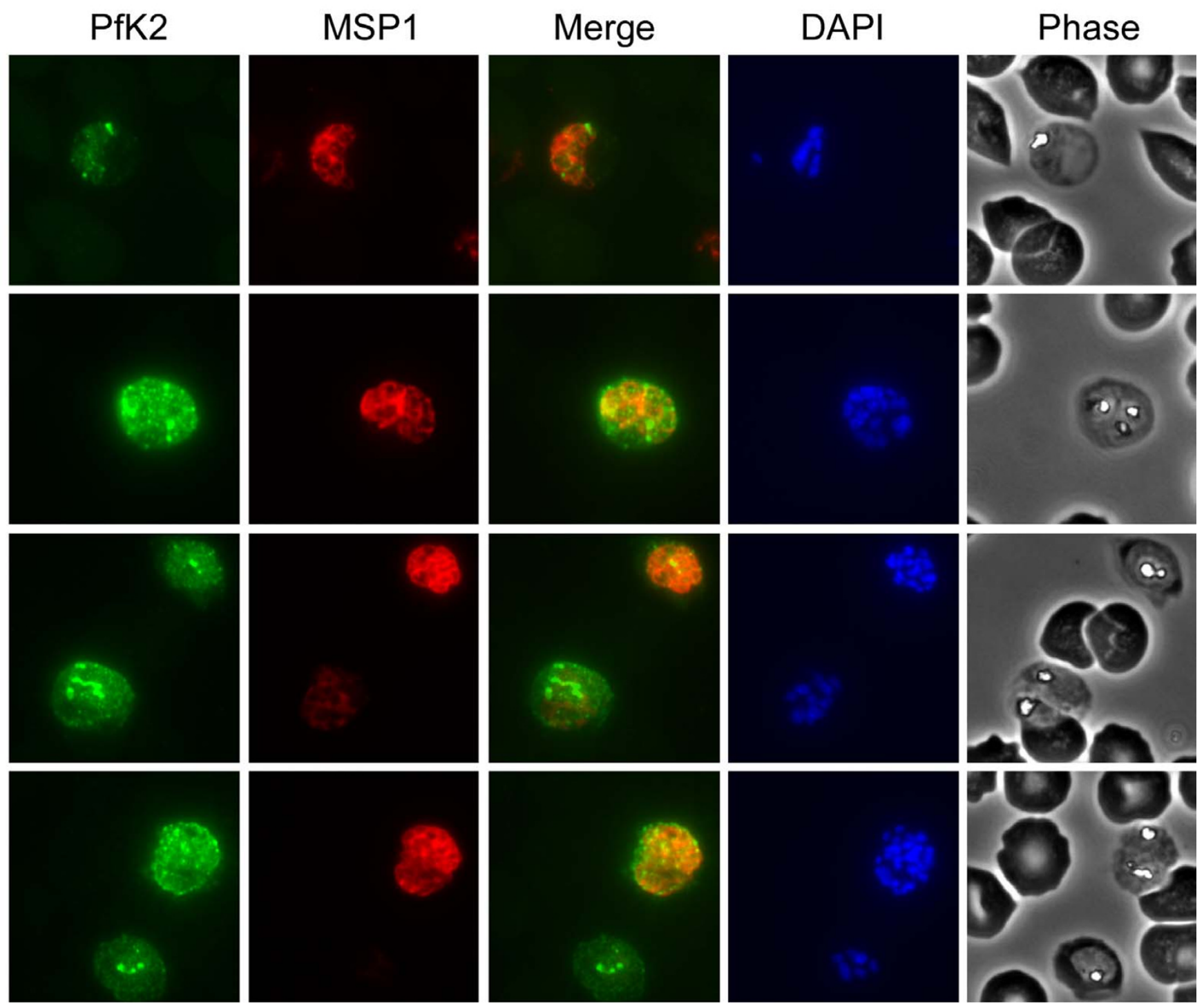

Figure 4

Localization of PfK2 in schizont-infected RBCs. Indirect Immunofluorescence Assays (IFAs) were performed using affinity purified rabbit anti-PfK2 F4 antibodies (green), and mouse monoclonal anti-MSPI (red). Individual and merged PfK2 and MSPI IFA panels are shown, in addition to the corresponding DAPI-labeled and phase images. Schizonts showed intense foci of labeling for PfK2, which appeared to correspond with the location of the developing merozoites within RBCs. In some cases, less intense PfK2 labeling was observed outside of the merozoites and in the RBC cytosol. 
plasma membrane is primarily generated by an electrogenic proton pump [21]. The proton efflux in this study was balanced by influx of $\mathrm{K}^{+}$, presumably through such surface channels. This $\mathrm{K}^{+}$transport may be carried by PfK1 and/or PfK2. During blood-stage development, various parasite proteins are synthesized and exported into the $\mathrm{RBC}$ resulting in modification of normal RBC structure and function [20]. New permeation pathways (NPP) are hypothesized to facilitate the transport of low molecular mass solutes $[2,3]$ from the RBC membrane to the parasite and are likely to include many of the predicted parasiteencoded channels and transporters encoded in the parasite genome [1].

$\mathrm{K}^{+}$channels are essential for the viability of all cells and are likely to be critical for malaria viability. The P. falciparum 3D7 genome encodes only two putative $\mathrm{K}^{+}$channel genes, $p f k 1$ and $p f k 2$. The search criteria employed in this study specified that the selectivity filter motif be flanked by at least two predicted membrane-spanning segments. Although the number of transmembrane domains can vary with $\mathrm{K}^{+}$channel type, in all cases, a single ion channel is created by the assembly of a combination of subunits that contribute four inner helices and four selectivity filter domains. Voltage-gated and $\mathrm{Ca}^{2+}$-activated $\mathrm{K}^{+}$channels contain six (or seven, in the case of big conductance (BK) $\mathrm{K}^{+}$channel) membrane spanning segments with the ion selectivity filter between the $5^{\text {th }}$ and $6^{\text {th }}$ membrane segment. Both PfK1 and PfK2 are predicted to have six membrane-spanning domains, and a pore region between the $5^{\text {th }}$ and $6^{\text {th }}$ segments based on hydropathy analysis and sequence alignments (Figure 1). The predicted pore-forming structures of PfK1 and PfK2 most resemble those of $\mathrm{Ca}^{2+}$-activated $\mathrm{K}^{+}$channels. PfK1 also has a cluster of evenly spaced arginines in the $4^{\text {th }}$ membrane-spanning segment that suggests the possibility of voltage-sensitivity. PfK2 lacks these charged residues in the $4^{\text {th }}$ transmembrane segment and is thus more akin to intermediate conductance (IK) or small conductance (SK) channels. Full protein sequence alignments of PfK1 and PfK2 with other characterized $\mathrm{K}^{+}$channels show less sequence similarity outside of the conserved pore-forming regions (data not shown), however their overall topologies are consistent with other voltage-gated and $\mathrm{Ca}^{2+}$-activated $\mathrm{K}^{+}$channels.

A possible third $P$. falciparum $\mathrm{K}^{+}$channel gene, PF14_0342, has been suggested from searches detailed by Martin et al [1]. This sequence is unlikely to encode a $\mathrm{K}^{+}$ channel due its lack of several key features that are common to many $\mathrm{K}^{+}$channels. The predicted selectivity filter contains a GKG motif instead of GYG (Fig. 1C) and lacks appropriate flanking transmembrane regions.

Here, the differential expression and localization of two putative $P$. falciparum potassium channels have been dem- onstrated. Recent global gene expression studies show pfk1 mRNA detectable throughout the entire 48-hour asexual cycle [17-19,22]. In support of these data, PfK1 was detected by immunoblot in all RBC stages, though more protein was detected in mature forms. By IFA, PfK1 expression was detected from early trophozoites through schizont stage parasites. In ring stage parasites, the density of PfK1 may be too low, such that its detection was beyond the sensitivity of IFA. More intense labeling in mature-iRBCs (Figure 3) and also in multiply-iRBCs was consistently observed.

Like KARHP and MESA, PfK1 localizes to the RBC membrane. It is hypothesized that PfK1 is expressed by the parasite and exported into the $\mathrm{RBC}$, where it is inserted into the RBC plasma membrane. Recent bioinformatic analyses of trafficked protein sequences have identified a conserved PEXEL/VTS motif that targets proteins for trafficking into the RBC $[23,24]$, in addition to the SS motif that directs proteins via the ER into the secretory pathway. In silico analysis $[24,25]$ of the PfK1 protein sequence for motifs indicated as potential export/transport signals failed to identify a VTS-like sequence, however manual searches yielded two potential VTS sequences, FFYRKLKNTFM and NFRRFLSSYKS (located 99 and 169 residues from the N-terminus, respectively). PfK1 also contains a predicted SS sequence (YDKRFSSRIKPK), located upstream of the VTS motifs and 20 residues from the $\mathrm{N}$ terminus of PfK1 (K. Haldar, personal communication).

PfK2 is primarily expressed in late schizonts and merozoites, in agreement with previous gene expression studies $[18,19,23]$. By IFA, PfK2 was detected in some, but not all, young ring stage parasites. Manual analysis of the PfK2 sequence revealed the presence of a possible SS cleavage motif and a low value VTS motif, but their configuration and location within the protein make it unclear whether these could function in the export of PfK2 (K. Haldar, personal communication).

From its localization, it is hypothesized that PfK1 encodes a parasite-induced ion channel that results in modification of the normal RBC to facilitate intracellular survival and maturation of the parasite by virtue of regulating $\mathrm{K}^{+}$ flux across the iRBC membrane, thereby effecting maintenance of the membrane potential and electrochemical gradient across the RBC plasma membrane and composition of the RBC cytosol.

PfK2, which is expressed predominantly in the merozoite, may function in the maintenance of membrane potential and the electrochemical gradient across merozoite membranes that are exposed to the human circulatory system. It is possible that PfK2 may act in concert with PfK1 dur- 


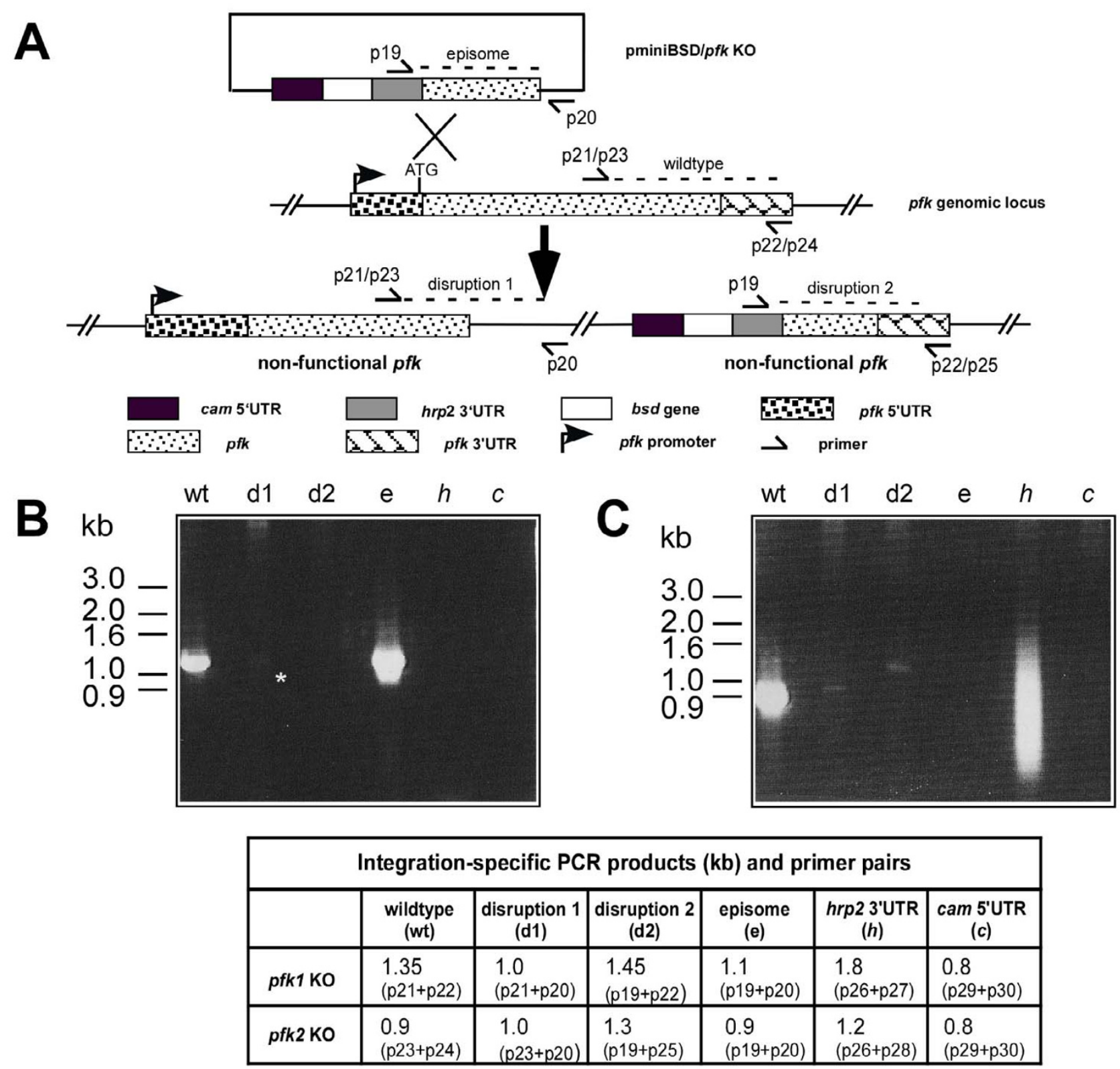

Figure 5

$p f k I$ and $p f k 2$ knockout transfections. A. Schematic representation of the knockout transfection plasmid (pminiBSD/pfk $\mathrm{KO}), p f k$ loci within the parasite genome, and the resultant $p f k$ loci after successful integration. Relative locations of primers used in the PCR detection of integration are shown. Integration-specific PCR products of $p f k I$ (B.) and pfk2 (C.) are shown. Expected sizes of PCR products resulting from each primer pair are shown in the table below. A $\sim 1.0 \mathrm{~kb}$ product $(*)$ was detected in $\mathrm{dl}$, indicative of integration into $\mathrm{p} f \mathrm{kl}$ (panel B), whereas $1.0 \mathrm{~kb}(\mathrm{dl})$ and $\mathrm{I} .3 \mathrm{~kb}(\mathrm{~d} 2)$ products indicative of integration into pfk2 (panel C) were detected. Integration into the genes $\mathrm{hrp2}$ and cam was also assessed, as regulatory elements of these Plasmodium sp. genes drive expression of the bsd gene present in the drug selection cassette on the plasmid. Integration into the $h r p 2$ loci was detected in the pfk2 transfection culture only (panel C, lane $h$ ).

ing this time to adapt to environment changes the parasites are exposed to when released from the rupturing RBC. Invasion studies using the Apicomplexan parasite
Toxoplasma gondii have shown that host cell invasion is accompanied by fluctuations of intracellular $\mathrm{Ca}^{2+}[26,27]$, similar to those described during antigen-stimulated acti- 
vation and proliferation of T cells. In those cells, effective $\mathrm{Ca}^{2+}$ signaling and ultimately downstream gene expression are modified by $\mathrm{K}^{+}$channel activity to maintain the cell membrane potential [28]. By analogy, it is hypothesized that PfK2 is a merozoite-specific $\mathrm{K}^{+}$channel that functions during the process of merozoite invasion of RBCs, perhaps functioning in ways to similarly effect intracellular $\mathrm{Ca}^{2+}$ signaling and gene transcription in the invading merozoite.

Heterologous expression and patch-clamp studies are required to definitively assign PfK1 and PfK2 to a class of $\mathrm{K}^{+}$channels. Functional expression of both native and codon-optimized PfK1 and PfK2 cDNAs in a variety of heterologous systems has so far proven unsuccessful (McBride and McDonald, unpublished data). Expression of active parasite-encoded $\mathrm{K}^{+}$channels in heterologous systems would illuminate the potential function of PfK1 and PfK2. Such a system could be adapted for the screening of novel specific blockers in a high through-put format for identification of possible new anti-malarials.

To examine the importance of these $\mathrm{K}^{+}$channels for parasite survival, attempts were made to knockout $p f k 1$ and $p f k 2$ expression. The ability to detect 'snap shots' of integration by PCR and inability to isolate parasite clones despite repeated attempts are consistent with the hypothesis that both $p f k 1$ and $p f k 2$ are critical for parasite viability. Failed attempts to disrupt various parasite genes thought to be necessary for parasite invasion, viability or propagation have been reported [10,29-31]. Further experimentation using either specific gene expression knockdown [10] or tetracycline-dependant conditional expression systems [32] may help unravel the precise functional roles of PfK1 and PfK2 in the regulation of $\mathrm{K}^{+}$ physiology in malaria-iRBCs.

\section{Abbreviations}

$\mathrm{K}^{+}$, Potassium Ion; RBC, Red Blood Cell; IFA, Indirect Immunofluorescence Assay; PfK, Plasmodium falciparum Potassium channel; PCR, polymerase chain reaction; GST, Glutathione S-transferase; PBS, Phosphate Buffered Saline; KAHRP, Knob-Associated Histidine Rich Protein; GRP(BiP), Glucose-Regulated Protein (Binding protein); BSA, Bovine Serum Albumin; DAPI, 4',6-Diamidino-2phenylinodole dihydrochloride; MESA, Mature parasiteinfected Erythrocyte Surface Antigen; ER, Endoplasmic Reticulum; iRBCs, infected Red Blood Cells; uRBCs, Uninfected Red Blood Cells; KO, Knockout; NPP, New Permeation Pathways; SK, IK and BK, Small, Intermediate and Big Conductance $\mathrm{K}^{+}$channels, respectively; PEXEL, Plasmodium Export Element; VTS, Vaculolar Transport Signal.

\section{Authors' contributions}

KLW performed the molecular biological analyses, antibody experiments, parasite culture, transfections and analyses, and writing of the manuscript. SMM performed the functional expression experiments. KK was involved in the initial in silico analysis, in guidance of the entire project and writing of the manuscript. TVM performed the in silico analysis, initial molecular cloning, and directed the project and writing of the manuscript. All authors read and approved the final manuscript.

\section{Acknowledgements}

We thank Ji-Mee Hwang and Danmei Qin for assistance during this investigation. We thank MR4 (Malaria Research and Reference Reagent Resource, ATCC) for providing the anti-GRP(BiP) and anti-MSPI antibodies (contributed by John $\mathrm{H}$. Adams and David C. Kaslow, respectively), Ross L. Coppel for providing the anti-KAHRP and anti-MESA antibodies and the Analytical Imaging Facility at Albert Einstein College of Medicine for their expert technical assistance and use of their imaging facilities. KLW is supported by an Australian NHMRC Howard Florey Centenary Research Fellowship. This research was funded by the USAMRMC grant \#DAMD 17-02-I-0290.

\section{References}

I. Martin RE, Henry RI, Abbey JL, Clements JD, Kirk K: The 'permeome' of the malaria parasite: an overview of the membrane transport proteins of Plasmodium falciparum. Genome Biol 2005, 6(3):R26

2. Kirk K: Membrane transport in the malaria-infected erythrocyte. Physiol Rev 200I, 8 I(2):495-537.

3. Kirk K: Channels and transporters as drug targets in the Plasmodium-infected erythrocyte. Acta Trop 2004, 89(3):285-298.

4. Gulbis JM, Doyle DA: Potassium channel structures: do they conform? Curr Opin Struct Biol 2004, I4(4):440-446.

5. Trager W, Jensen JB: Human malaria parasites in continuous culture. Science 1976, 193:673-675.

6. PlasmoDB: The Plasmodium genome resource. [http:// www.plasmodb.org]

7. Lakshmanan V, Bray PG, Verdier-Pinard D, Johnson DJ, Horrocks P, Muhle RA, Alakpa GE, Hughes RH, Ward SA, Krogstad DJ, Sidhu AB, Fidock DA: A critical role for PfCRT K76T in Plasmodium falciparum verapamil-reversible chloroquine resistance. Embo J 2005, 24( ( 3):2294-2305.

8. Fidock DA, Nomura T, Wellems TE: Cycloguanil and its parent compound proguanil demonstrate distinct activities against Plasmodium falciparum malaria parasites transformed with human dihydrofolate reductase. Mol Pharmacol 1998 , 54(6): I | 40- I I 47

9. Sidhu $A B$, Verdier-Pinard D, Fidock DA: Chloroquine resistance in Plasmodium falciparum malaria parasites conferred by pfcrt mutations. Science 2002, 298(559I):210-2I3.

10. Waller KL, Muhle RA, Ursos LM, Horrocks P, Verdier-Pinard D, Sidhu AB, Fujioka $H$, Roepe PD, Fidock DA: Chloroquine resistance modulated in vitro by expression levels of the Plasmodium falciparum chloroquine resistance transporter. The Journal of biological chemistry 2003, 278(35):33593-3360I.

II. Bennett B], Mohandas N, Coppel RL: Defining the minimal domain of the Plasmodium falciparum protein MESA involved in the interaction with the red cell membrane skeletal protein 4.I. J Biol Chem 1997, 272(24):I5299-I5306.

12. Lambros C, Vanderberg JP: Synchronization of Plasmodium falciparum erythrocytic stages in culture. J Parasitol 1979, 65(3):4l 8-420.

13. Soulie S, Moller JV, Falson P, le Maire M: Urea reduces the aggregation of membrane proteins on sodium dodecyl sulfatepolyacrylamide gel electrophoresis. Analytical biochemistry 1996, 236(2):363-364.

14. Sweadner KJ: Trypsin inhibitor paradoxically stabilizes trypsin activity in sodium dodecyl sulfate, facilitating proteolytic fingerprinting. Analytical biochemistry I99I, 194(I): I30-135. 
15. Kumar N, Koski G, Harada M, Aikawa M, Zheng H: Induction and localization of Plasmodium falciparum stress proteins related to the heat shock protein 70 family. Mol Biochem Parasitol I99I, 48(I):47-58.

16. Ellekvist $\mathrm{P}$, Ricke $\mathrm{CH}$, Litman $\mathrm{T}$, Salanti A, Colding $\mathrm{H}$, Zeuthen $\mathrm{T}$, Klaerke DA: Molecular cloning of a $\mathrm{K}(+)$ channel from the malaria parasite Plasmodium falciparum. Biochem Biophys Res Commun 2004, 3 18(2):477-484.

17. Ben Mamoun C, Gluzman IY, Hott C, MacMillan SK, Amarakone AS, Anderson DL, Carlton JM, Dame JB, Chakrabarti D, Martin RK, Brownstein BH, Goldberg DE: Co-ordinated programme of gene expression during asexual intraerythrocytic development of the human malaria parasite Plasmodium falciparum revealed by microarray analysis. Mol Microbiol 200I, 39(I):26-36.

18. Le Roch KG, Johnson JR, Florens L, Zhou Y, Santrosyan A, Grainger M, Yan SF, Williamson KC, Holder AA, Carucci DJ, Yates JR 3rd, Winzeler EA: Global analysis of transcript and protein levels across the Plasmodium falciparum life cycle. Genome Res 2004, I 4(I I):2308-23I8.

19. Bozdech Z, Llinas M, Pulliam BL, Wong ED, Zhu J, DeRisi JL: The transcriptome of the intraerythrocytic developmental cycle of Plasmodium falciparum. PLoS Biol 2003, I(I): E5.

20. Cooke BM, Mohandas N, Coppel RL: The malaria-infected red blood cell: structural and functional changes. Adv Parasitol 200I, 50: I-86

21. Allen RJ, Kirk K: The membrane potential of the intraerythrocytic malaria parasite Plasmodium falciparum. The Journal of biological chemistry 2004, 279( I 2): I I 264- I I 272.

22. Young JA, Fivelman QL, Blair PL, de la Vega P, Le Roch KG, Zhou Y, Carucci DJ, Baker DA, Winzeler EA: The Plasmodium falciparum sexual development transcriptome: a microarray analysis using ontology-based pattern identification. Mol Biochem Parasitol 2005, I 43(I):67-79.

23. Marti M, Good RT, Rug M, Knuepfer E, Cowman AF: Targeting malaria virulence and remodeling proteins to the host erythrocyte. Science 2004, 306(5703): 1930-1933.

24. Hiller NL, Bhattacharjee S, van Ooij C, Liolios K, Harrison T, LopezEstrano C, Haldar K: A host-targeting signal in virulence proteins reveals a secretome in malarial infection. Science 2004 306(5703): 1934-1937.

25. Lopez-Estrano C, Bhattacharjee S, Harrison T, Haldar K: Cooperative domains define a unique host cell-targeting signal in Plasmodium falciparum-infected erythrocytes. Proc Natl Acad Sci U S A 2003, I00(2I): I2402-12407.

26. Lovett JL, Marchesini N, Moreno SN, Sibley LD: Toxoplasma gondii microneme secretion involves intracellular $\mathrm{Ca}(2+)$ release from inositol 1,4,5-triphosphate (IP(3))/ryanodinesensitive stores. The Journal of biological chemistry 2002, 277(29):25870-25876.

27. Lovett JL, Sibley LD: Intracellular calcium stores in Toxoplasma gondii govern invasion of host cells. J Cell Sci 2003, I I6(Pt 14):3009-3016.

28. Panyi G: Biophysical and pharmacological aspects of $\mathbf{K}+$ channels in T lymphocytes. Eur Biophys J 2005, 34(6):5 I 5-529.

29. Triglia T, Healer J, Caruana SR, Hodder AN, Anders RF, Crabb BS, Cowman AF: Apical membrane antigen I plays a central role in erythrocyte invasion by Plasmodium species. Mol Microbiol 2000, 38(4):706-718.

30. Cowman AF, Baldi DL, Duraisingh M, Healer J, Mills KE, O'Donnell RA, Thompson J, Triglia T, Wickham ME, Crabb BS: Functional analysis of Plasmodium falciparum merozoite antigens: implications for erythrocyte invasion and vaccine development. Philos Trans R Soc Lond B Biol Sci 2002, 357( I 4I 7):25-33.

31. Cowman AF, Crabb BS: Invasion of red blood cells by malaria parasites. Cell 2006, I 24(4):755-766.

32. Meissner M, Krejany E, Gilson PR, de Koning-Ward TF, Soldati D, Crabb BS: Tetracycline analogue-regulated transgene expression in Plasmodium falciparum blood stages using Toxoplasma gondii transactivators. Proc Natl Acad Sci U S A 2005 102(8):2980-2985.

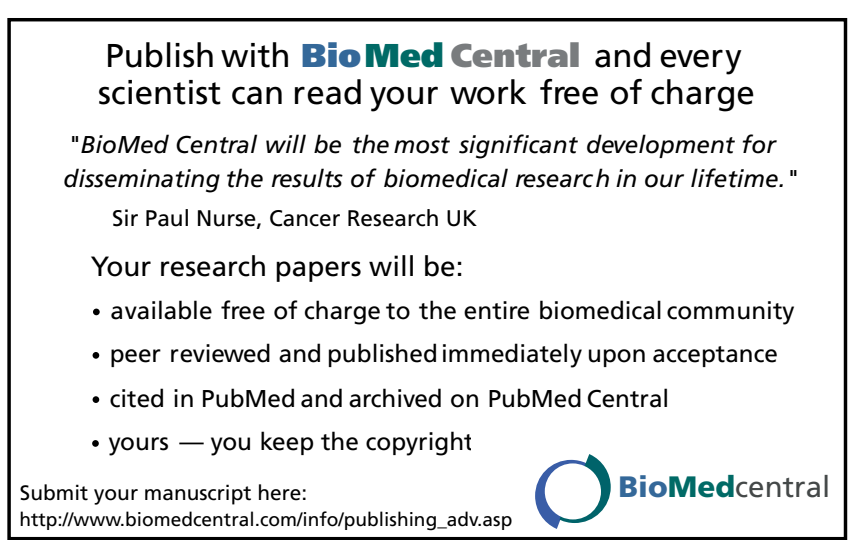

